\title{
Efficacy of two species of entomopathogenic fungi against the stored-grain pest, Sitophilus granarius L. (Curculionidae: Coleoptera), via oral ingestion
}

Yacoub Ahmad Batta

\begin{abstract}
Sitophilus granarius is a serious stored-grain insect that can destroy the whole grain of cereal crops. The present research demonstrated that two species of entomopathogenic fungi (EPF), Beauveria bassiana and Metarhizium anisoplidae, killed the treated adults of S. granarius when applied via oral ingestion. Mortality of S. granarius was shown by growth and development of the ingested conidia in the insect gut causing death of infected insects. During bioassays, the fungus conidia were mixed with wheat flour then provided to the insects for feeding on the mixture for $24 \mathrm{~h}$. The insects were surface-treated with a contact fungicide (Merpan $\left.{ }^{\circledR}\right)$ to kill the external conidia that could penetrate the cuticle and develop to kill the infected insects. Results indicated that the most effective ratio of mixing fungus conidia with the wheat flour was $0.1: 2.0(\mathrm{~g} / \mathrm{g})$ where this ratio should contain $2.75 \times 10^{8}$ conidia/g of M. anisopliae mixture or $2.6 \times 10^{8}$ conidia/g of B. bassiana mixture. Ingested conidia of each fungus caused a first mortality of $S$. granarius adults after 10 and 12 days of B. bassiana and $M$. anisopliae treatments, respectively. They also caused 50\% mortality after 2.787 and 3.047 weeks of treatments for the same fungi, respectively. The novelty of this research is in adjusting and applying a technique of disinfection of insect cuticle with a fungicide and then verifying the presence of applied fungi in the infected insects. The practical application of this method was discussed.
\end{abstract}

Keywords: Biocontrol, Sitophilus granaries, Beauveria bassiana, Metarhizium anisoplidae, Surface sterilization

\section{Background}

Entomopathogenic fungi (EPF) are potential biocontrol agents of many insect pest species (Batta 2007; Gabarty et al. 2014 and Han et al. 2014). One of the main groups of agricultural insect pests that can be effectively controlled by EPF is the stored-grain insects (Batta 2016). For examples, the yellow meal worm, Tenebrio molitor L. (Batta et al. 2010); confused flour beetle, Tribolium confusum Jacquelin du Val.; corn weevil, Sitophilus zeamais Motschulsky and lesser grain borer, Rhyzopertha dominica Fabricius (Barra et al. 2013); rice weevil,

Correspondence: yabatta@najah.edu

Laboratory of Plant Protection, Department of Plant Production and

Protection, Faculty of Agriculture and Veterinary Medicine, An-Najah National University, Nablus, West Bank, Palestine
Sitophilus oryzae L. (Kavallieratos et al. 2014) and rice meal moth, Corcyra cephalonica Stainton (Kaur et al. 2014). The mode of action of these fungi is through germination of conidia that adheres to the outer surface of target insect cuticle producing germ tube that can penetrate the cuticle towards the insect hemocoel. Penetrating germ tube multiplies forming mycelium in the interior portion causing eventually death of the infected insect (Pedrini et al. 2007; Zhang et al. 2008; Stephou et al. 2012; Ortiz-Urquiza and Keyhani 2013). On the cadavers of dead insects due to infection with EPF, the internal mycelium penetrates the cuticle to the outside then grows on the cuticle surface producing large quantity of conidia at favorable conditions of temperature and relative humidity (Fig. 1a, b). The efficacy of applied 

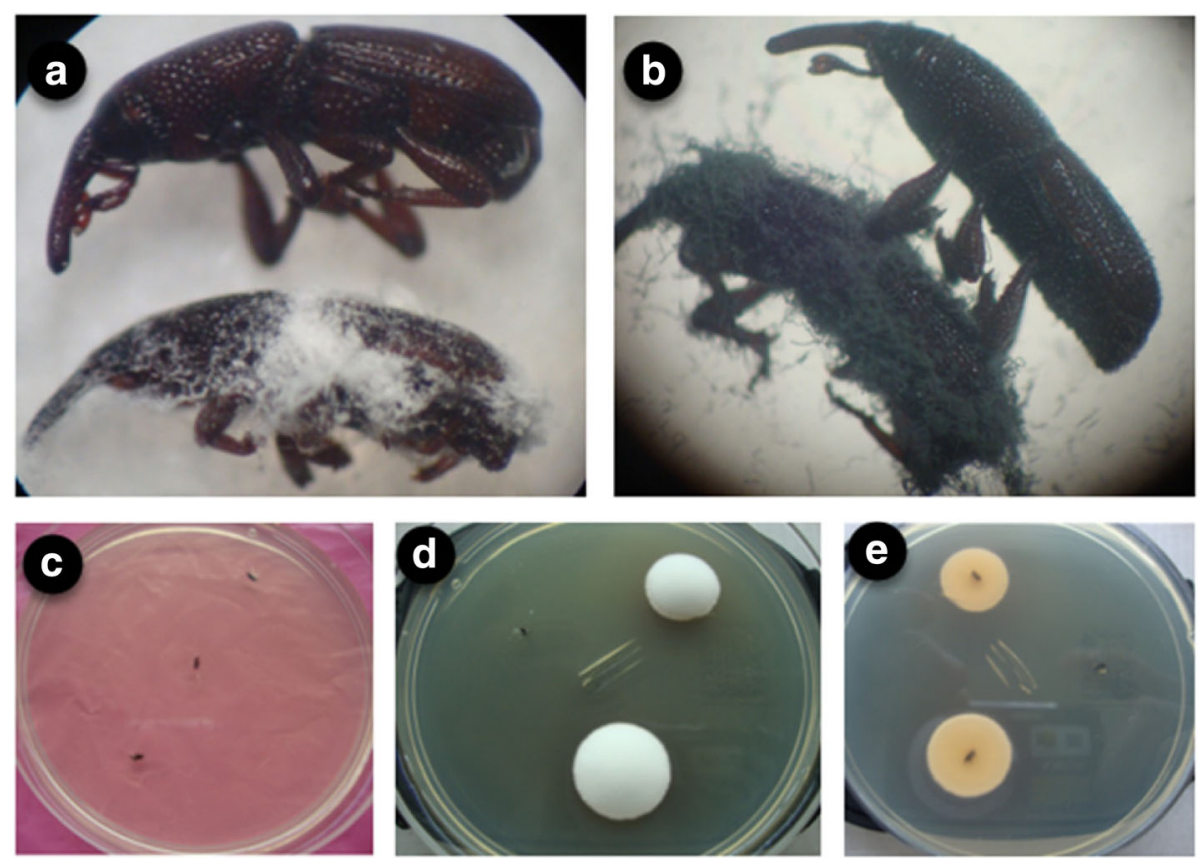

Fig. 1 Infection and isolation of two species of EPF from adults of Sitophilus granarius (strain SG5), a Infection of S. granarius adult (lower picture) with Beauveria bassiana (strain BB2) exhibited by white superficial mycelium growth, upper picture is uninfected or healthy. $\mathbf{b}$ Infection of $S$. granarius' adults (lower picture) with Metarhizium anisopliae (strain MA1) exhibited by olive green superficial mycelium growth, upper picture is uninfected or healthy. $\mathbf{c}$ Isolation technique of ingested conidia of B. bassiana or M. anisopliae from treated adults of S. granarius after being superficially disinfected then plated using a selective medium (SDA + Chloramphenicol). d-e Growth of B. bassiana on the plate surface with a selective medium (SDA + Chloramphenicol) (upper and lower views of fungus growth as a white dense mycelia growth on the plate's surface in case of positive isolation)

EPF depends on many factors, especially the susceptibility of insect species and virulence of the strain or isolate of EPF (Michalaki et al. 2006; Ahmed 2010 and Han et al. 2014).

To the best of our knowledge, there were no reports about the effect of EPF on stored-grain insects via oral ingestion. Therefore, the general objective of the present study is to demonstrate if the EPF conidia incorporated via oral ingestion by $S$. granarius adults could be able to generate mortality, whereas the specific objectives are the following: (i) to develop an effective technique for a superficial disinfection of EPF fungus conidia that may adhere to the outer surfaces of insect cuticle then germinate and penetrate into the hemocoel, (ii) to develop an effective technique of isolation for testing the viability of ingested conidia or its developing mycelium thus showing viability of these fungal structures, (iii) since the death of infected insects is an indication on viability of ingested conidia, the mortality of treated insects will be taken as an indication of the effectiveness of applied technique, and (iv) to prepare mixtures of fungus conidia of EPF and wheat flour then feed them to the target insects to ensure that the ingested conidia will kill the infected insects.

\section{Methods}

Isolates of Metarhizium anisopliae and Beauveria bassiana MA1 and BB2 are the isolates of M. anisopliae and $B$. bassiana that were used, respectively, in the conducted bioassays. They were originally isolated from infected adults and larvae of the ground beetle, Carabus nemoralis L. (Carabidae: Coleoptera). The isolation was done, at first, on a selective medium: Sabouraud Dextrose Agar (SDA) + Chloramphenicol $(250 \mathrm{mg} / \mathrm{L})$ then, pure cultures of these isolates were obtained from monosporic cultures on the same culture medium.

\section{Strain of Sitophilus granarius}

Male and female adults of S. granarius (strain SG5) were used in the conducted bioassays. They were obtained from insect cultures kept in the insectary of Faculty of Agriculture and Veterinary Medicine, An-Najah National University, Nablus, Palestinian Territories. S. granarius was reared on wheat and rice grain in plastic pots, covered with pieces of cheese cloth to permit a good aeration during the rearing process. 


\section{Mixtures of fungal isolates and wheat flour}

Mixtures of conidia of each isolate (MA1 and BB2) and wheat flour were prepared. This is because, during their growth on the culture medium, $M$. anisopliae and $B$. bassiana form a dry dense crust of conidia on the medium surface within 2 weeks of incubation at $20{ }^{\circ} \mathrm{C}$ and $12 \mathrm{~h}$ of light period. The crust which was olivegreen in M. anisopliae and white in B. bassiana could be easily removed from the surface of culture plate by scraping them, using a sterile scalpel. The scraped conidia could be then thoroughly mixed with wheat flour (as a palatable commodity for stored-grain insects) in order to be used for feeding the adults of $S$. granarius. Mixtures of dry fungus conidia and wheat flour were prepared, using different ratios of both ingredients as the following: T1 $(0.2 \mathrm{~g}$ of fungus conidia mixed with 2 . $0 \mathrm{~g}$ of wheat flour or 0.2:2.0 ratio, $\mathrm{g} / \mathrm{g})$, T2 (0.1 g of fungus conidia to $2.0 \mathrm{~g}$ of flour or 0.1:2.0 ratio, $\mathrm{g} / \mathrm{g}$ ), $\mathrm{T} 3$ (0.05 g of fungus conidia to $2.0 \mathrm{~g}$ of flour or $0.05: 2.0$ ratio $\mathrm{g} / \mathrm{g}$ ), and T4 ( $0 \mathrm{~g}$ of fungus conidia to $2.0 \mathrm{~g}$ of flour or $0: 2.0$ ratio, $\mathrm{g} / \mathrm{g}$ ). The last ratio which had only wheat flour substrate was used as a control treatment for testing the effect of the substrate. The prepared mixture with the highest ratio of fungus conidia and wheat flour (T1) contained $\left(5.5 \times 10^{8}\right.$ conidia/g of $M$. anisopliae mixture and $5.2 \times 10^{8}$ conidia/g of $B$. bassiana mixture), whereas the other mixtures $\mathrm{T} 2$ and $\mathrm{T} 3$ of the two fungi were prepared by making successive dilutions of the highest ratio by a factor of one half, respectively. T4 was used as a control since it contains only the substrate (flour). The concentration of fungus conidia in the highest ratio mixture (T1) was measured by suspending and homogenizing $1 \mathrm{~g}$ of the mixture in $50 \mathrm{ml}$ of sterile distilled water then counting the conidial number in the suspension using a hemocytometer.

\section{Bioassays}

Two grams of each one of the above-mentioned mixtures: T1, T2, T3, T4, were transferred into small plastic pots $(10 \mathrm{~cm}$ diameter and $7 \mathrm{~cm}$ depth), and then 20 adults of S. granarius (strain SG5) were introduced into each pot. Four pots, representing four replicates, were used in each treatment (mixture type). Sixteen pots were used, in total, in bioassays conducted for each EPF species. Each pot was covered with a piece of cheese cloth as described before. The insects were left for feeding on the contents of each mixture for $24 \mathrm{~h}$, then the insects, in each replicate, were separated immediately and treated superficially with a contact fungicide such as Merpan $^{\circ}$ (active ingredient Captan, concentration $800 \mathrm{~g} /$ $\mathrm{kg}$ of wettable granular formulation). An aqueous suspension containing $0.2 \% \operatorname{Merpan}^{\circ}(w / v)$ was prepared and then used for the superficial treatment so the application rate of the fungicide was $2.0 \mathrm{~g} / \mathrm{L}(w / v)$. The treatment was done by spraying the insects with the fungicide suspension, using ULV spraying technique in a closed chamber. The fungicide was used as a secondary agent to prevent the conidial germination of $M$. anisopliae or B. bassiana on insect cuticle. Target conidia in this treatment may come from the mixtures used for feeding insects. However, the ingested conidia during feeding were not affected by the fungicide treatment. Treated insects with the fungicide were then placed in new plastic pots similar to the previous ones but containing $10 \mathrm{~g}$ of healthy rice grain each then incubated in a growth chamber at $20^{\circ} \mathrm{C}$ and $12 \mathrm{~h}$ of light period for 7 weeks. Briefly, after the feeding period and the transfer of insects, each pot contained 20 treated adults of $S$. granarius and $10 \mathrm{~g}$ of healthy rice grain and covered with a piece of cheese cloth as described before. The rate of application was calculated at $20 \mathrm{~g}$ of mixture per kilogram of grain. Each treatment was repeated three times to confirm the reproducibility of the results.

\section{Evaluation of the treatment efficacy}

To evaluate the efficiency of the fungicide treatment and to demonstrate the viability of ingested conidia, the following techniques and procedures were applied: (i) testing the viability of ingested conidia by S. granarius adults after feeding on the mixtures described above by isolating the ingested fungi on a selective culture medium (SDA + Chloramphenicol $250 \mathrm{mg} / \mathrm{L}$ ) within the period of incubation (6 to 7 weeks) so a positive or negative isolation of fungi could be obtained and (ii) counting the dead $S$. granarius adults/replicate/treatment over a period of 7 weeks, following superficial treatment, where the mortality percentage was calculated and then used for plotting the linear relationship between the time post treatments and mortality percentage.

To ensure that the mortality of insects observed within the period of 7 weeks of incubation was caused by the effect of ingested conidia of tested fungi, the following plating technique of isolation was applied: (i) the dead insects resulted from feeding on mixtures of fungus conidia and wheat flour were first superficially disinfected by dipping them in a solution of $2.0 \%$ sodium hypochlorite for $30 \mathrm{~s}$ to exclude any bacterial contamination or mold growing during fungal growth on culture medium, (ii) the insects were rinsed three times with sterile distilled water to wash the residues of disinfecting solution then left for drying up under aseptic conditions, and then (iii) the air-dried insects were aseptically placed in plates with selective medium (SDA + Chloramphenicol $250 \mathrm{mg} / \mathrm{L}$ ) (Fig. 1c). This is for testing the viability of ingested conidia or mycelium developing from these conidia. Therefore, a positive or negative isolation was recorded, according to the growth of these fungi on the 
culture medium within 10 days of incubation of plates at $20{ }^{\circ} \mathrm{C}$ and $12 \mathrm{~h}$ light per day.

\section{Statistical analyses}

One-way ANOVA was carried out to test significant differences between the means of mortality percentage of treated $S$. granarius adults by $M$. anisopliae after 7 weeks of treatment or by B. bassiana after 6 weeks of treatment, using different mixtures of fungus conidia and wheat flour. Scheffe test was used for comparison of the abovementioned means. Data obtained on mortality percentage of $S$. granarius by the two fungi were also used for plotting a linear relationship between the mortality percentage and the time of incubation after treatments, using different ratios of fungus conidia and wheat flour. Regression equations and $R^{2}$ values for each ratio used were calculated and provided with its corresponding regression line. Comparisons of regression lines were made according to the regression analysis.

\section{Results and discussion}

Isolation of ingested EPF from S. granarius adults treated superficially with Merpan ${ }^{\circledast}$

Isolation of ingested conidia or its developing mycelium from S. granarius adults fed on mixtures of EPF conidia + wheat flour and treated with Merpan ${ }^{\circ}$ was positive, if the fungus mycelium grows on the surface of culture plates (Fig. 1d, e). For the mixtures of $M$. anisopliae conidia + wheat flour, positive isolations were obtained starting from the third day, following the feeding time for treatments $\mathrm{T} 1, \mathrm{~T} 2$, and $\mathrm{T} 3$, until the first death of treated adults on 12th day for $\mathrm{T} 1$ and 13th day for $\mathrm{T} 2$ and T3 (Table 1). For the mixtures of B. bassiana conidia + wheat flour, the isolations were also positive starting from the third day, following the feeding time for treatments T1, T2, and T3 until the first death of treated adults on 10th day for T1, 11th day for T2, and 12th for T3 (Table 2). For untreated S. granarius adults with Merpan', the isolation of M. anisopliae was positive from the second day, following the feeding time for treatments T1, T2, and T3, until the first death of treated adults on the seventh day for T1, eighth day for T2, and ninth day for T3 (Table 1). For B. bassiana, the isolation was also positive starting the second day, following the feeding time for the treatments $\mathrm{T} 1, \mathrm{~T} 2$, and T3 until the first death of treated adults on the fifth day for T1, seventh day for T2, and eighth for $\mathrm{T} 3$ (Table 2). The applied technique demonstrated its effectiveness by inhibiting the germination and penetration of external EPF conidia existing on insect surface, but it did not affect the conidia ingested by insects so that we can reveal the presence of internal, hemocoelic fungus vegetative cells, as well as any potentially viable conidia in the insect alimentary tracts. No previous studies are available on the lethal effect of ingested conidia of EPF in the stored-grain insects, but few earlier studies are available on other insect species that indicated the possibility of such effect in the nymphs of the grasshopper, Melanoplus sanguinipes F., when fed on bait substrate of B. bassiana (Inglis et al. 1996).

\section{Mortality of S. granarius adults fed on conidia of EPF}

Results indicated that $S$. granarius adults, fed on M. anisopliae or B. bassiana conidia mixtures + wheat flour, died due to ingesting the conidia of the tested fungi. This effect was shown by the daily counting of dead

Table 1 Isolation of ingested fungus conidia (or mycelium developing from them) of Metarhizium anisopliae from infected adults of granary weevil, Sitophilus granarius, after feeding them on mixtures of M. anisopliae conidia + wheat flour for $24 \mathrm{~h}$ then incubated after surface treatment with Merpan ${ }^{\oplus}$ on healthy rice grains at $20^{\circ} \mathrm{C}$

\begin{tabular}{|c|c|c|c|c|c|c|c|c|}
\hline \multirow{3}{*}{$\begin{array}{l}\text { Time (days) } \\
\text { for isolation } \\
\text { the fungus }\end{array}$} & \multicolumn{8}{|c|}{ Mixture ratios of M. anisopliae conidia and wheat flour $(w / W)^{a}$} \\
\hline & \multicolumn{2}{|l|}{$\mathrm{T} 1(0.2: 2.0 \mathrm{~g} / \mathrm{g})$} & \multicolumn{2}{|l|}{ T2 $(0.1: 2.0 \mathrm{~g} / \mathrm{g})$} & \multicolumn{2}{|l|}{ T3 $(0.05: 2.0 \mathrm{~g} / \mathrm{g})$} & \multicolumn{2}{|c|}{$\mathrm{T} 4(0: 2.0 \mathrm{~g} / \mathrm{g})$} \\
\hline & Treated $^{b}$ & Untreated $^{b}$ & Treated $^{\mathrm{b}}$ & Untreated $^{b}$ & Treated $^{\mathrm{b}}$ & Untreated $^{\mathrm{b}}$ & Treated $^{b}$ & Untreated $^{\mathrm{b}}$ \\
\hline 1 day & $-{ }^{d}$ & $-{ }^{\mathrm{d}}$ & $-^{d}$ & $-{ }^{d}$ & $-^{d}$ & $-{ }^{d}$ & $-{ }^{d}$ & $-^{d}$ \\
\hline 2 days & - & + & - & + & - & + & - & - \\
\hline 3 days & + & + & + & + & + & + & - & - \\
\hline 7 days & + & + (first death) & + & + & + & + & - & - \\
\hline 8 days & + & & + & + (first death) & + & + & - & - \\
\hline 9 days & + & & + & & + & + (first death) & - & - \\
\hline 12 days & + (first death) & & + & & + & & - & - \\
\hline 13 days & & & + (first death) & & + (first death) & & - & - \\
\hline
\end{tabular}

${ }^{\mathrm{a}}$ Four replicates were used per treatment and each replicate contains 20 adults of S. granarius

${ }^{\mathrm{b}}$ Treated or untreated adults of S. oryzae with the contact fungicide Merpan ${ }^{\circledast}$

'Time in days from treatment with Merpan ${ }^{\circledR}$ to starting of fungus isolation on a selective culture medium

${ }^{d}+$ means positive isolation of the fungus on a selective culture medium (Sabouraud Dextrose Agar + Chloramphenicol 250 mg/L); - means negative isolation of the fungus 
Table 2 Isolation of ingested fungus conidia (or mycelium developing from them) of Beauveria bassiana from infected adults of granary weevil, Sitophilus granarius, after feeding them on mixtures of B. bassiana conidia + wheat flour for $24 \mathrm{~h}$ then incubated after surface treatment with Merpan ${ }^{\circledast}$ on healthy rice grains at $20^{\circ} \mathrm{C}$

\begin{tabular}{|c|c|c|c|c|c|c|c|c|}
\hline \multirow{3}{*}{$\begin{array}{l}\text { Time (days) for } \\
\text { isolation of the } \\
\text { fungus }^{c}\end{array}$} & \multicolumn{8}{|c|}{ Mixture ratios of $B$. bassiana conidia and wheat flour $(w / W)^{a}$} \\
\hline & \multicolumn{2}{|l|}{$\mathrm{T} 1(0.2: 2.0 \mathrm{~g} / \mathrm{g})$} & \multicolumn{2}{|l|}{$\mathrm{T} 2(0.1: 2.0 \mathrm{~g} / \mathrm{g})$} & \multicolumn{2}{|c|}{$\mathrm{T} 3(0.05: 2.0 \mathrm{~g} / \mathrm{g})$} & \multicolumn{2}{|c|}{$\mathrm{T} 4(0: 2.0 \mathrm{~g} / \mathrm{g})$} \\
\hline & Treated $^{\mathrm{b}}$ & Untreated $^{b}$ & Treated $^{\mathrm{b}}$ & Untreated $^{b}$ & Treated $^{\mathrm{b}}$ & Untreated $^{\mathrm{b}}$ & Treated $^{b}$ & Untreated $^{b}$ \\
\hline 1 day & $-^{d}$ & $-^{d}$ & $-{ }^{d}$ & $-^{d}$ & $-^{d}$ & $-{ }^{d}$ & $--^{d}$ & $-{ }^{d}$ \\
\hline 2 days & - & + & - & + & - & + & - & - \\
\hline 3 days & + & + & + & + & + & + & - & - \\
\hline 5 days & + & + (first death) & + & + & + & + & - & - \\
\hline 7 days & + & & + & + (first death) & + & + & - & - \\
\hline 8 days & + & & + & & + & + (first death) & - & - \\
\hline 10 days & + (first death) & & + & & + & & - & - \\
\hline 11 days & & & + (first death) & & + & & - & - \\
\hline 12 days & & & & & + (first death) & & - & - \\
\hline
\end{tabular}

${ }^{\mathrm{a}}$ Four replicates were used per treatment and each replicate contains 20 adults of S. granarius

${ }^{\mathrm{b}}$ Treated or untreated adults of S. oryzae with the contact fungicide Merpan ${ }^{\odot}$

${ }^{\mathrm{C}}$ Time in days from treatment with Merpan ${ }^{\circledast}$ to starting of fungus isolation on a selective culture medium

$d_{+}$means positive isolation of the fungus on a selective culture medium (Sabouraud Dextrose Agar + Chloramphenicol 250 mg/L); - means negative isolation of the fungus

insects over a period of incubation after feeding that extended up to 7 weeks for M. anisopliae (Fig. 3) and up to 6 weeks for B. bassiana (Fig. 4). Mixtures of conidia of the two fungi with wheat flour at ratios $0.2: 2.0$ and 0 . 1:2.0 $(w / w)$ caused $100 \%$ mortality in treated adults while mixture at ratio 0.05:2.0 $(w / w)$ caused mortality of $50 \%$ for M. anisopliae and 55\% for B. bassiana (Fig. 2). Mortality rates in the control treatments were 9 and $10 \%$ for $M$. anisopliae and B. bassiana, respectively (Fig. 2). Statistical analysis indicated that there were significant differences among the means of mortality percentages in the different mixtures after 7 weeks of the treatment for $M$. anisopliae $[F$ value $=11.874$ (sig. 0 . $001), \mathrm{df}=2$ and 12 and subset for alpha <0.01] and 6 weeks of the treatment for $B$. bassiana $[F$ value $=21.000$ (sig. 0.001), $\mathrm{df}=2$ and 12 and subset for alpha $<0.01$ ]. By comparing the above means to the control mean, significant differences $(P<0.05)$ were obtained, especially with the mixtures of $0.2: 2.0$ or 0.1:2.0; therefore, these mixtures are considered the most effective ones with insignificant
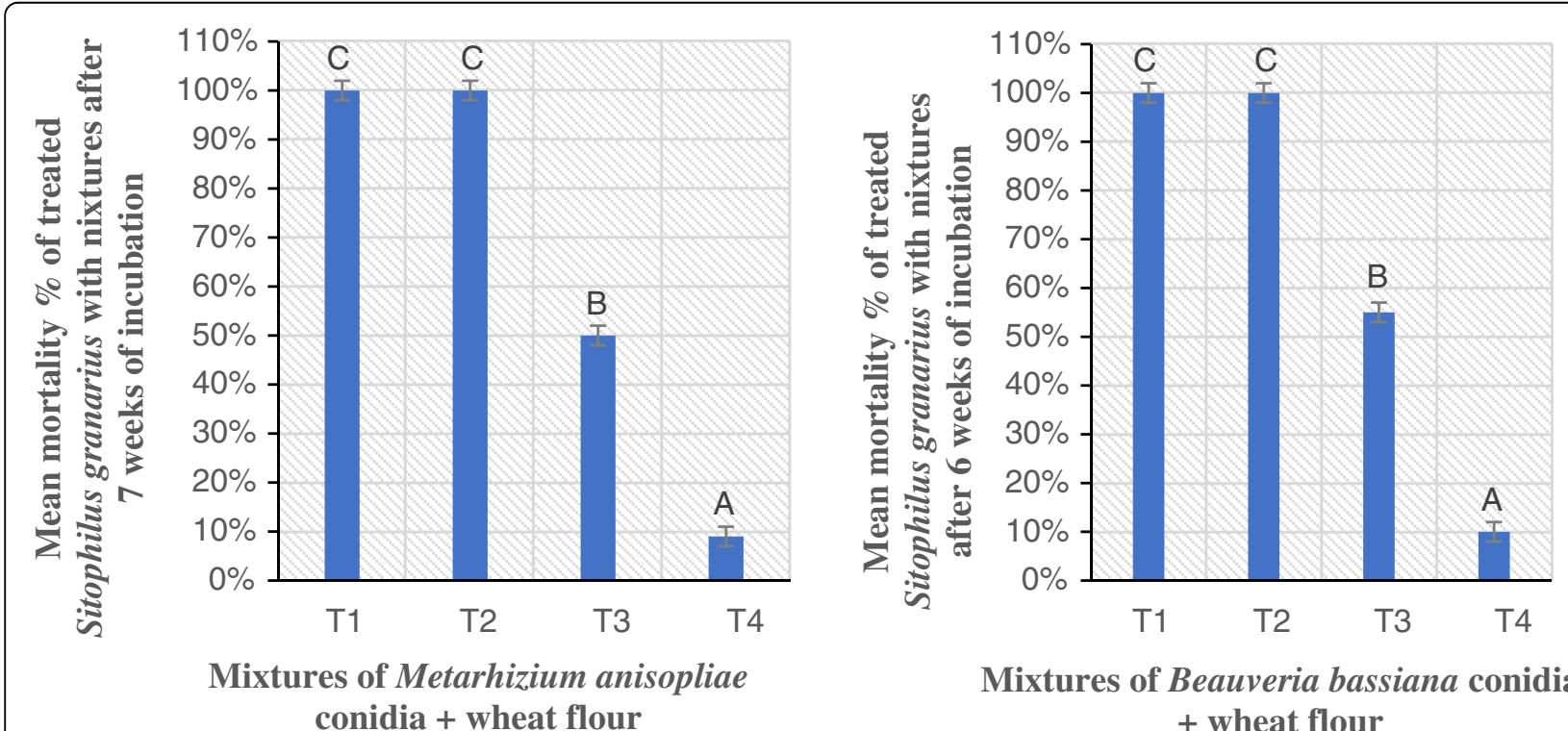

Mixtures of Beauveria bassiana conidia + wheat flour

Fig. 2 Mortality of Sitophilus granarius adults. Means followed by the same letter are insignificantly different at $P=0.05$ using ANOVA and Scheffe for means comparison. Ratios of mixtures $(w / w)$ are $T 1=0.2: 2.0 ; T 2=0.1: 2.0 ; T 3=0.05: 2.0 ; T 4=0: 2.0$ 
differences. The mixture T2 (ratio 0.1:2.0) of both species of EPF was recommended to be used for the control of $S$. granarius adults. This mixture should be adjusted during preparation to contain $2.75 \times 10^{8}$ conidia/g of mixture for $M$. anisopliae (one half of the highest concentration for this species in the mixture of T1: $\left.5.5 \times 10^{8}\right)$ and $2.6 \times 10^{8}$ conidia/g of mixture for $B$. bassiana (one half of the highest concentration for this species in the mixture T1: 5 . $2 \times 10^{8}$ ). The application rate of the recommended mixture was calculated at $20 \mathrm{~g} / \mathrm{kg}$ grain. Regression analysis of the data indicated that the calculated regression equations and $R^{2}$ values of the mixtures of $M$. anisopliae conidia + wheat flour were as follows: for T1, $y$ $=14.405 x+0.8333, R^{2}=0.9019 ; \mathrm{T} 2, y=15.179 x+3.75$, $R^{2}=0.9595 ; \mathrm{T} 3, y=6.5476 x+2.0833, R^{2}=0.7703 ; \mathrm{T} 4, y=$ $3.035 x+0.9141, R^{2}=0.6352$ (Fig. 3), whereas that of $B$. bassiana, they were as follows: for T1, $y=14.821 x+3$. 3929, $R^{2}=0.8348$; T2, $y=16.786 x+3.2143, R^{2}=0.9208$; T3, $y=8.3929 x+0.5357, R^{2}=0.7784$; T4, $y=2.3214 x+0$. 8929, $R^{2}=0.5633$ (Fig. 4). Based on the regression equation of the recommended mixture (T2) in both fungi indicated above, the time needed for killing $50 \%$ of treated adults of S. granarius was calculated at 3.047 weeks for $M$. anisopliae and 2.787 weeks for B. bassiana.
Results indicated that the mortality rate of adults of $S$. granarius was caused by the development of ingested conidia; the evidence on this conidial development was the positive isolation of these fungi from the internal portion of treated insects by plating method, using a selective culture medium (SDA + Chloramphenicol) (Fig. 1d, e). However, the way by which the ingested conidia develop inside the insect's gut and kill the host insects is not known exactly, probably that these conidia germinate in the gut, then the germ tube of germinated conidia penetrates the gut walls to enter the haemocoel where they can grow profusely and kill the infected insect but this explanation needs to be proved or demonstrated in future studies. Until present, no reports are available on the effect of ingested conidia of EPF in killing the stored-grain insects. However, earlier studies revealed similar results but in other insect species. For example, Kramm and West (1982) reported that plating hindgut contents of workers of termites (Reticulitermes sp.) that were exposed to Metarhizium, Gliocladium, and Beauveria on culture medium indicated the presence of living conidia in their gut, following their ingestion. Also, Lacey et al. (1988) indicated that viable conidia of $M$. anisopliae were detected in the gut of Culex quinquefasciatus larvae that

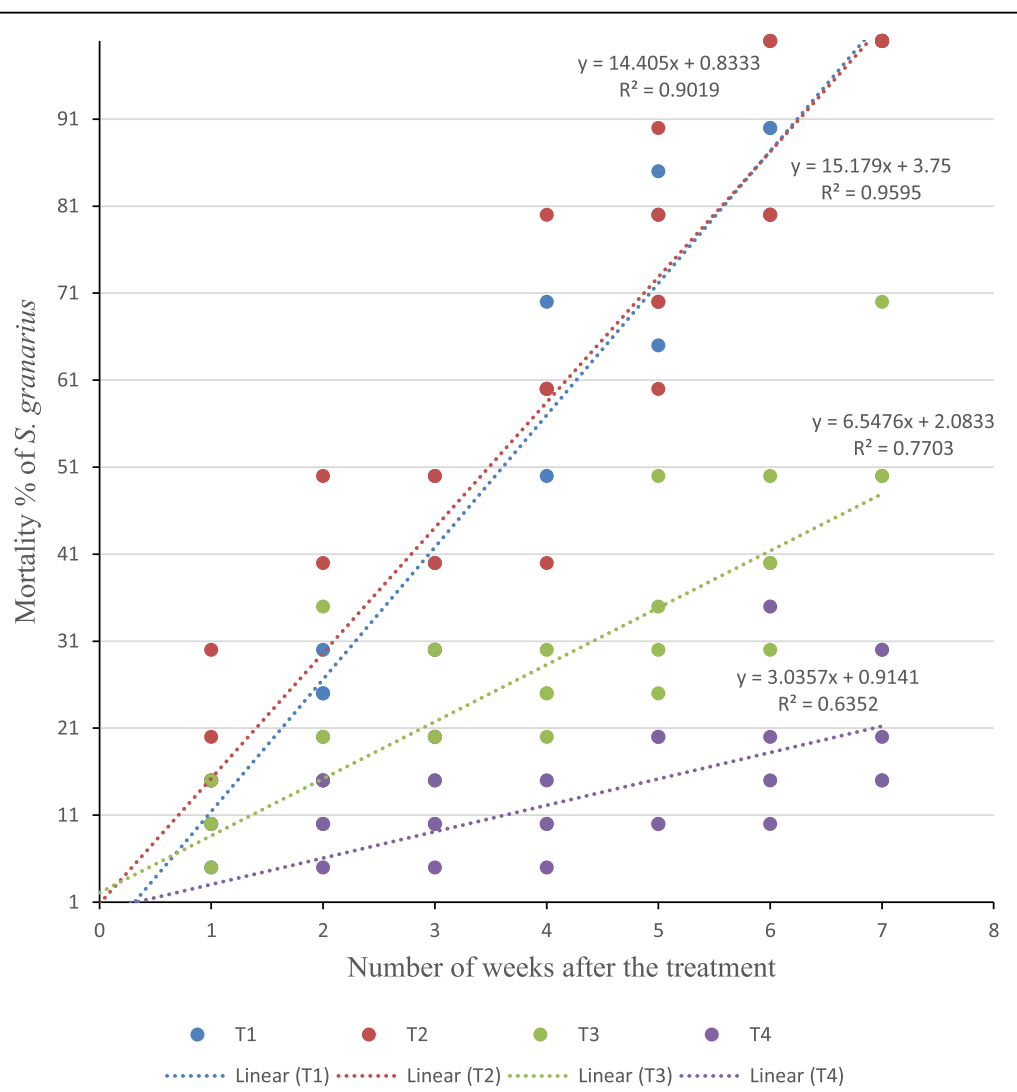

Fig. 3 Mortality of adults of granary weevils (Sitophilus granarius; strain SG5) as a result feeding on mixtures of Metarhizium anisopliae (strain MA1) conidia + wheat flour for $24 \mathrm{~h}$. Ratios of mixtures $(\mathrm{g} / \mathrm{g})$ are $\mathrm{T} 1=0.2: 2.0 ; \mathrm{T} 2=0.1: 2.0 ; \mathrm{T} 3=0.05: 2.0 ; \mathrm{T} 4=0: 2.0$ 


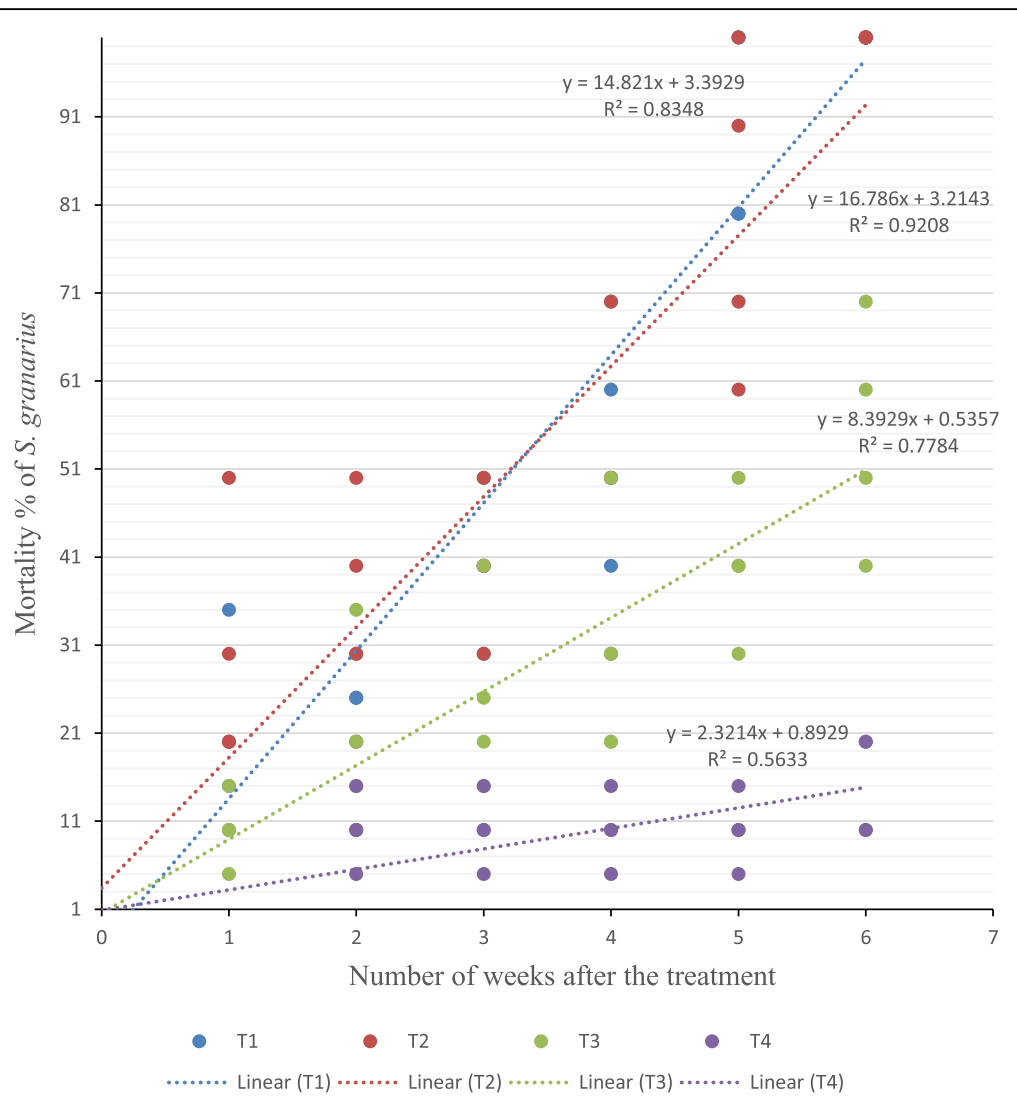

Fig. 4 Mortality of adults of the granary weevils (Sitophilus granarius; strain SG5) as a result feeding on mixtures of Beauveria bassiana (strain BB2) conidia + wheat flour for $24 \mathrm{~h}$. Ratios of mixtures $(\mathrm{g} / \mathrm{g})$ are $\mathrm{T} 1=0.2: 2.0 ; \mathrm{T} 2=0.1: 2.0 ; \mathrm{T} 3=0.05: 2.0 ; \mathrm{T} 4=0: 2.0$

were fed on detergent-wetted conidia. Moreover, Miranpuri and Khachatourians (1991) found that the most preferred site for fungal development of B. bassiana after ingestion by the larvae of Aedes aegypti was the larval gut and germination of the ingested conidia and their development in the gut caused the death of host larvae. Viable conidia were also recovered in the frass of grasshopper nymphs Melanoplus sanguinipes after ingestion of lettuce and bran substrates inoculated with $B$. bassiana conidia (Inglis et al. 1996).

It is important to mention that recent reports are available on the effect of endophytic EPF that live internally in the plants such as $M$. anisopliae that lives in Brassica napus and practices control effect against the larvae of Plutella xylostella (Batta 2013), B. bassiana in Vitis vinifera that exercises adverse effect against various sucking insects (Rondot and Reineke 2017), and B. bassiana in durum wheat plants that exercises reducing effect against Spodoptera littoralis (Sanchez-Rodriguez et al. 2017). Therefore, the lethal effect of these endophytic fungi on target insects is shown by oral ingestion of plant sap in sucking insects or by eating the plant parts in chewing insects. The sap or plant parts that contain these fungi affect target insect by oral ingestion but not by cuticular penetration, so these fungi can play an important role in the control of insects that attack host plants of these fungi.

It was observed that the development of mycelium resulted from ingested conidia until the death of hosting insects of $S$. granarius was slow in comparison to the development of the mycelium of the same tested fungi that resulted from germination and penetration of external conidia through the insect cuticle. This is because our results indicated that $100 \%$ mortality of adults of $S$. granarius was achieved within 6 to 7 weeks after the treatment, due to application of mixtures of conidia of M. anisopliae or B. bassiana with wheat flour compared to (93.3\%) mortality of adults of Rhyzopertha domnica, within 1 week due to the cuticular penetration by external germinated conidia applied by spraying the insects with conidial suspension of M. anisopliae (Batta 2005). Although a long period of time was needed to achieve $100 \%$ mortality in the treated S. granarius adults, the applied method is characterized by the following traits: easy to prepare and apply, cheap since it uses a cheap substrate of wheat flour as a carrier of the fungal conidia, and it can be used in grain stores by mixing the grain with flour substrate carrying the fungus conidia, in 
addition to the possibility of transmission of infection from one generation of target insects to another where the first infections are usually produced by the first introduction of fungi through sporulation of cadavers.

From a practical point of view, the purpose of using the above-mentioned technique is to be an alternative mean to the chemical control of target insects infesting grains in the stores. For using the technique in grain stores, the following steps are recommended to be done: (i) preparing the mixture of fungus conidia and wheat flour at the recommended ratio $(0.1: 2.0 \mathrm{w} / \mathrm{w}$, respectively) giving that this mixture should contain $2.75 \times 10^{8}$ conidia/g of mixture for $M$. anisopliae and $2.6 \times 10^{8}$ conidia/g of mixture for $B$. bassiana, and (ii) distributing the prepared mixture thoroughly in the store when it is empty or by mixing it with the grain using the recommended application rate of $20 \mathrm{~g}$ of mixture per $1 \mathrm{~kg}$ of grain.

\section{Conclusions}

A technique for disinfecting the insect cuticle with a fungicide and verifying the presence of EPF in infected host insects after feeding on a substrate containing these fungi was described and applied. The growth and development of these fungi after ingestion caused death of infected insects. This would be a newly recorded interaction between this type of specific host and associated pathogens. Possible application of the results in pest management programs of the target stored-grain insect as an alternative mean to chemical control was discussed.

\section{Acknowledgements \\ I would like to thank my colleagues in the Department of Plant Production and Protection, An-Najah National University who helped in editing the English language of the manuscript.}

\section{Competing of interests}

The author declares that he/she has no competing interests.

\section{Authors' contributions}

The author carried out the bioassays then collected the data and performed the statistical analysis. The author prepared the manuscript and revised it then read and approved the final version.

\section{Ethics approval and consent to participate}

Not applicable.

\section{Publisher's Note}

Springer Nature remains neutral with regard to jurisdictional claims in published maps and institutional affiliations.

Received: 3 January 2018 Accepted: 27 April 2018

Published online: 15 May 2018

\section{References}

Ahmed BI (2010) Potentials of entomopathogenic fungi in controlling the menace of maize weevil Sitophilus zeamais Motsch (Coleoptera: Curculionidae) on stored maize grain. Arch Phytopathol Plant Protec 43:107-115

Barra P, Rosso L, Nesci A, Etcheverry M (2013) Isolation and identification of entomopathogenic fungi and their evaluation against Tribolium confusum,
Sitophilus zeamais, and Rhyzopertha dominica in stored maize. J Pest Sci 86: $217-226$

Batta Y (2007) Biocontrol of almond bark beetle (Scolytus amygdali GeurinMeneville, Coleoptera: Scolytidae) using Beauveria bassiana (Bals.) Vuill. (Deuteromycotina: Hyphomycetes). J Appl Microbiol 103:1406-1414

Batta Y (2013) Efficacy of endophytic and applied Metarhizium anisopliae (Metch.) Sorokin (Ascomycota: Hypocreales) against larvae of Plutella xylostella L. (Yponomeutidae: Lepidoptera) infesting Brassica napus plants. Crop Protec 44:128-134

Batta Y, Murdoch G, Mansfield S (2010) Investigations into the formulation and application of entomopathogenic fungi against larvae of yellow meal worm (Tenebrio molitor L., Coleoptera: Tenebrionidae). General Appl Entomol 39:5-8

Batta YA (2005) Control of the lesser grain borer (Rhyzopertha dominica (F.), Coleoptera: Bostrichidae) by treatments with residual formulations of Metarhizium anisopliae (Metschnikoff) Sorokin (Deuteromycotina: Hyphomycetes). J Stored Products Res 41:221-229

Batta YA (2016) Recent advances in formulation and application of entomopathogenic fungi for biocontrol of stored grain insects. Biocont Sci Technol 26:1171-1183

Gabarty A, Salem HM, Fouda M, Abas A, Ibrahim AA (2014) Pathogenicity induced by the entomopathogenic fungi Beauveria bassiana and Metarhizium anisopliae in Agrotis ipsilon. J Radiol Res Appl Sci 7:95-100

Han JH, Jin BR, Kim JJ, Lee SY (2014) Virulence of entomopathogenic fungi Metarhizium anisopliae and Paecilomyces fumosoroseus for the microbia control of Spodoptera exigua. Biological Cont 42:385-390

Inglis DG, Johnson DL, Goettel MS (1996) Effect of bait substrate and formulation on infection of grasshopper nymphs by Beauveria bassiana. Biocont Sc Technol 6:35-50

Kaur S, Thakur A, Rajput M (2014) A laboratory assessment of the potential of Beauveria bassiana (Balsamo) Vuillemin as a biocontrol agent of Corcyra cephalonica Stainton (Lepidoptera: Pyrallidae). J Stored Products Res 59:185-189

Kavallieratos NG, Athanassiou CG, Aountala MM, Kontodimas DC (2014) Evaluation of the entomopathogenic fungi Beauveria bassiana, Metarhizium anisopliae, and Isaria fumosorosea for control of Sitophilus oryzae. J Food Protec 1:4-17

Kramm KR, West DF (1982) Termite pathogens: effects of ingested Metarhizium, beauveria, and Gliocladium conidia on worker termites (Reticulitermes sp.). $J$ Invertebr Pathol 40:7-11

Lacey CM, Lacey CL, Roberts RD (1988) Route of invasion and histopathology of infection with Metarhizium anisopliae in Culex quinquefasciatus. J Invertebr Pathol 52:108-118

Michalaki M, Athanassiou C, Kavallieratos N, Batta Y, Balotis G (2006) Effectiveness of Metarhizium anisopliae (Metchinkoff) Sorokin applied alone or in combination with diatomaceous earth against Tribolium confusum (Du Val) larvae: influence of temperature, relative humidity and type of commodity. Crop Protec 25:418-425

Miranpuri GS, Khachatourians GG (1991) Infection sites of the entomopathogenic fungus Beauveria bassiana in the larvae of the mosquito Aedes aegypti. Entomol Experiment Appl 59:19-27

Ortiz-Urquiza A, Keyhani NO (2013) Action on the surface: entomopathogenic fungi versus the insect cuticle. Insects 4:357-374

Pedrini N, Crespo R, Juárez MP (2007) Biochemistry of insect epicuticle degradation by entomopathogenic fungi. Compar Biochem Physiol C: Toxicol Pharmacol 146:124-137

Rondot Y, Reineke A (2017) Endophytic Beauveria bassiana in grapevine Vitis vinifera (L.) reduces infestation with piercing-sucking insects. AGRIS (in press, corrected proof)

Sanchez-Rodriguez AR, Diaz SR, Zamarreno AM, Quesada E (2017) An endophytic Beauveria bassiana strain increases spike production in bread and durum wheat plants and effectively controls cotton leafworm (Spodoptera littoralis) larvae. Biological Contr (in press, corrected proof)

Stephou VK, Tjamos SE, Paplomatas EJ, Athanassiou CG (2012) Transformation and attachment of Beauveria bassiana conidia on the cuticle of Tribolium confusum and Sitophilus oryzae in conjunction with diatomaceous earth. J Pest Sci 85:387-394

Zhang YJ, Feng MG, Fan YH, Luo ZB, Yang XY, Wu D, Pei YA (2008) Cuticledegrading protease (CDEP-1) of Beauveria bassiana enhances virulence. Biocontr Sci Technol 18:551-563 